\title{
Canonical description of ideal magnetohydrodynamic flows and integrals of motion.
}

\author{
A. V. KATS \\ Usikov Institute for Radiophysics and Electronics National Academy of Sciences \\ of Ukraine, 61085, 12 Ak. Proskury St., Kharkiv, Ukraine \\ Fax: +380 (572) 44-11-05 e-mail: avkats@akfirst.kharkiv.com; avkats@ire.kharkov.ua
}

(Dated: 9.06.03)

\begin{abstract}
In the framework of the variational principle the canonical variables describing magnetohydrodynamic (MHD) flows of general type (i.e., with spatially varying entropy and nonzero values of all topological invariants) are introduced. It is shown that the velocity representation of the Clebsch type following from the variational principle with constraints is equivalent to that resulting from the generalization of the Weber transformation performed in the paper for the case of arbitrary MHD flows. Using such complete velocity representation enables us not only to describe the general type flows in terms of single-valued functions, but also to solve the intriguing problem of the "missing" MHD integrals of motion. The set of hitherto known MHD local invariants and integrals of motion appears to be incomplete: for the vanishing magnetic field it does not reduce to the set of the conventional hydrodynamic invariants. And if the analogs of the vorticity and helicity were discussed earlier for the particular cases, the analog of Ertel invariant has been so far unknown. It is shown that all "missing" invariants are expressed in terms of the decomposition of the velocity representation into the "hydrodynamic" and "magnetic" parts. In spite of the nonunique character of such representation it is shown that there exists a natural restriction of the gauge transformations set allowing one to make the invariants gauge independent. It is found that on the basis of the new invariants introduced a wide set of high-order invariants can be constructed. The new invariants are relevant both for the deeper insight into the problem of the topological structure of the MHD flows as a whole and for the examination of the stability problems. The additional advantage of the proposed approach is that it enables one to deal with discontinuous flows, including all types of possible breaks.
\end{abstract}

PACS numbers: $04.20 \mathrm{Fy}, 47.10 .+\mathrm{g}, 47.65$

\section{INTRODUCTION.}

It is well-known that description of the solid media flows in terms of the canonical (Hamiltonian) variables is very useful and effective, see for instance [1, 2]. On the basis of the Hamiltonian variables it is possible to deal with all nonlinear processes in unified terms which are independent of the specific problem related to the media under investigation. For instance, all variants of the perturbation theory are expressed in terms of different order nonlinear vertices, which along with the linear dispersion relation contain the specific information relating to the concrete system, cf. Refs. 3, 4]. In studying nonlinear stability problems the conventional Hamiltonian approach based upon the corresponding variational principle allows one to use the Hamiltonian along with other integrals of motion (momentum, number of quasi-particles, topological invariants) in order to construct the relevant Lyapunov functional, cf. Refs. [5, 6, 7, 8, 9]. Therefore, it is important to address the problem of introducing the canonical variables and corresponding variational principle for the general type MHD flows (i. e., rotational, non-barotropic and including all types of breaks that are possible for MHD) and obtaining the complete set of the local invariants, see definition and discussions in original papers [10, 11, 12, 13] and in the recent review [1]. As for the first item, the example of the variational principle describing all possible breaks is given in the recent work [14].

Here in the framework of some modification of the variational principle of the latter work we examine the problem of the MHD invariants. Note that the set of invariants for MHD discussed in the literature has been so far incomplete. The statement becomes apparent when it is considered that for the vanishing magnetic field this set has to be reduced to that of the conventional hydrodynamic invariants. But this limit transition does not reproduce Ertel, vorticity and helicity invariants existing for the hydrodynamic flows.

Despite the fact that for the dissipation-free MHD flows there exist additional topological invariants, namely, magnetic helicity and cross-helicity, introduced in the papers, [15, 16, 17], the analogs of the vorticity and helicity invariants have not been discussed with necessary completeness thus far, cf., for instance, the recent review [1]. The related quantities were mentioned for the specific cases of symmetric flows in the works 18, 19, 20, the vorticity and helicity invariants for the incompressible flows have been obtained recently in Refs. 8, 9]. But analog of the Ertel invariant have not been presented so far (see the short communication in 21] ). The problem of obtaining the analogs of the hydrodynamic invariants consists in the non-potential character of the Lorentz force. Therefore, the vorticity 
and helicity of the total velocity field $\mathbf{v}$ are not conserved along with the Ertel invariant construction, $\rho^{-1} \boldsymbol{\omega} \cdot \nabla s$. Nevertheless, corresponding generalizations have to exist, which becomes evident from the simple consideration. Namely, let us consider the well known set of invariants for dissipation-free MHD flows (energy, magnetic and cross helicity). Setting the magnetic field zero we arrive at zero values of the magnetic and cross helicity invariants, but do not get Ertel invariant (and hydrodynamic vorticity and helicity for the barotropic flows). This fact indicates incompleteness of the MHD invariants set. Evidently, there have to exist MHD analog of the Ertel invariant passing on to the hydrodynamic Ertel invariant for the vanishing magnetic field. Below we derive the MHD generalization for the Ertel invariant and show that the generalized vorticity and helicity invariants also exist for the compressible barotropic MHD flows. The possibility of obtaining these invariants is based upon the velocity decomposition in the two parts, "hydrodynamic" and "magnetic". The latter vanishes with the magnetic field vanishing and can be presented in the form of the vector product of the magnetic field and the canonically conjugate momentum and was first introduced in the paper 22]. In spite of the artificial character of the velocity field decomposition at first sight, we show that the decomposition naturally follows both from the least action principle in the canonical variables and from the partial integration of the Euler equations of motion (generalized Weber transformation, cf. Ref. 23, 24]). For the incompressible flows the latter was presented in the papers [8, 9]. Note that the "hydrodynamic" part of the velocity is of the Clebsch type but involves vector potentials instead of the scalar ones, see discussion in Refs. 1, 14, 25, 26. . The use of the vector Clebsch potentials allows one to deal with the flows possessing nontrivial topology, contrary to the restriction to the scalar potentials. If the latter are single-valued then the helicity vanishes identically.

The outline of the paper is as follows. In section $\amalg$ we briefly discuss the appropriate variational principle, introducing the Clebsch type velocity representation by means of constraints and defining the canonical variables. The velocity representation thus obtained yields the necessary decomposition. In section 11 we develop generalization of the Weber transformation and show that it leads to the velocity representation, which is equivalent to that following from the variational principle under discussion. In section IV we examine the MHD integrals of motion, introducing "missing" MHD invariants, and discuss their transformation properties relating to the gauge change. We show that there exist natural gauges under which the additional basic invariants become unambiguous, specifically that with a vanishing initial value of the magnetic part of the velocity representation. In section $\nabla$ we make some conclusions and formulate problems to be solved later.

\section{VARIATIONAL PRINCIPLE AND CANONICAL VARIABLES.}

Let us briefly describe the variational principle and subsidiary variables describing dissipation-free MHD. Starting with the standard Lagrangian density

$$
\mathcal{L}=\rho \frac{v^{2}}{2}-\rho \varepsilon(\rho, s)+\frac{\mathbf{H}^{2}}{8 \pi}
$$

where $\rho, s$ and $\varepsilon(\rho, s)$ are the fluid density, entropy and internal energy, respectively, $\mathbf{H}$ denotes the magnetic field, we have to include the constraint terms in the action $\mathcal{A}$. Then the action can be presented as

$$
\mathcal{A}=\int d t L^{\prime}, \quad L^{\prime}=\int d \mathbf{r} \mathcal{L}^{\prime}, \quad \mathcal{L}^{\prime}=\mathcal{L}+\mathcal{L}_{c}
$$

where $\mathcal{L}_{c}$ is the part of the Lagrangian density respective for the constraints,

$$
\mathcal{L}_{c}=\rho D \varphi+\lambda D \boldsymbol{\mu}+\sigma D s-\mathbf{M} \cdot\left(\frac{\partial \mathbf{A}}{\partial t}-\mathbf{v} \times \operatorname{curl} \mathbf{A}+\nabla \Lambda\right)-\frac{\mathbf{H} \cdot \operatorname{curl} \mathbf{A}}{4 \pi} .
$$

Here $D=\partial_{t}+\mathbf{v} \cdot \nabla$ is the substantial (material) derivative and $\mathbf{A}$ is the vector potential. 32 . Including the terms with $\Lambda$ and $\mathbf{H}=$ curlA into $\mathcal{L}_{c}$ allows us to obtain the dynamic equation for the vector potential in the gauge invariant form (see Eq. (10) below) and to introduce relation $\mathbf{H}=$ curlA strictly into the variational principle.

Supposing first that all variables introduced (including velocity) are independent, we obtain the following set of variational equations

$$
\begin{gathered}
\delta \varphi \Longrightarrow \partial_{t} \rho+\operatorname{div}(\rho \mathbf{v})=0, \\
\delta \rho \Longrightarrow \quad D \varphi=w-v^{2} / 2,
\end{gathered}
$$




$$
\begin{gathered}
\delta \boldsymbol{\lambda} \Longrightarrow \quad D \boldsymbol{\mu}=0 \\
\delta \mu_{m} \Longrightarrow \quad \partial_{t} \lambda_{m}+\operatorname{div}\left(\lambda_{m} \mathbf{v}\right)=0, \\
\delta \sigma \Longrightarrow \quad D s=0, \\
\delta s \Longrightarrow \quad \partial_{t} \sigma+\operatorname{div}(\sigma \mathbf{v})=-\rho T, \\
\delta \mathbf{M} \Longrightarrow \mathbf{d}=\operatorname{virl} \mathbf{A}-\nabla \Lambda, \\
\delta \mathbf{A} \Longrightarrow \quad \partial_{t} \mathbf{M}=\frac{\operatorname{curl} \mathbf{H}}{4 \pi}+\operatorname{curl}[\mathbf{v} \times \mathbf{M}] . \\
\delta \mathbf{H} \Longrightarrow \quad \mathbf{H}=\operatorname{curl} \mathbf{A}, \\
\delta \Lambda \Longrightarrow \quad \operatorname{div} \mathbf{M}=0,
\end{gathered}
$$

where $w$ and $T$ are the enthalpy density and temperature. 33

Note that in this section we assume the velocity field to be independent of other variables. Therefore, variation with respect to $\mathbf{v}$ results in the velocity representation

$$
\delta \mathbf{v} \Longrightarrow \rho \mathbf{v}=-\rho \nabla \varphi-\lambda_{m} \nabla \mu_{m}-\sigma \nabla s-\mathbf{H} \times \mathbf{M} .
$$

It is convenient to rewrite it in a shortened form that emphasizes its structure. Bearing in mind that the velocity potential $\varphi$, the vector Lagrange markers $\boldsymbol{\mu}$, entropy $s$ and the vector potential $\mathbf{A}$ can be treated as generalized coordinates, one can see that $\rho, \boldsymbol{\lambda}, \sigma$ and subsidiary field $-\mathbf{M}$ are conjugated momenta, respectively. Let $[4]$

$$
\mathcal{Q}=(Q, \mathbf{A}), \quad Q=(\varphi, \boldsymbol{\mu}, s), \quad \mathcal{P}=\delta \mathcal{A} / \delta\left(\partial_{t} \mathcal{Q}\right), \quad P=(\rho, \boldsymbol{\lambda}, \sigma), \quad \mathcal{P}=(P,-\mathbf{M}) .
$$

Then the velocity representation takes the transparent form

$$
\mathbf{v}=\mathbf{v}_{0}(\mathcal{P}, \nabla \mathcal{Q}), \quad \mathbf{v}_{0}=\mathbf{v}_{h}+\mathbf{v}_{M}, \quad \mathbf{v}_{h}=-\frac{P}{\rho} \nabla Q, \quad \mathbf{v}_{M}=-\frac{\mathbf{H} \times \mathbf{M}}{\rho} .
$$

Here subindexes $h$ and $M$ correspond to the "hydrodynamic" and "magnetic" parts of the velocity field. The suffix zero on $\mathbf{v}$ underlines the fact that $\mathbf{v}_{0}$ is supposed to be the dependent variable, it is expressed in terms of the canonical variables by means of the representation found. The hydrodynamic part, $\mathbf{v}_{h}$, corresponds to the generalized Clebsch representation, cf. [14, 25, 26], and the magnetic part, $\mathbf{v}_{M}$, coincides with the traditional term if we replace the divergence-free field $\mathbf{M}$ by curlS. This term was first introduced by Zakharov and Kuznetsov, cf. Ref. [22]. But they used the incomplete form for the hydrodynamic part of the velocity, restricting it to the scalar Clebsch variables. This reduced form [35] evidently corresponds to the flows with zero-valued generalized helicity (or, for $\mathbf{H} \rightarrow 0$, it results in the hydrodynamic helicity vanishing) if the scalar Clebsch variables are single-valued. Besides, the above velocity representation involves the entropy term, $-\sigma \nabla S / \rho$. The latter is essential for the discontinuous flows with any types of the dynamically allowable breaks, including shocks, cf. Refs. 14, 21, 25, 26]. Note that Lagrange markers $\boldsymbol{\mu}$ are continuous crossing the break surface, contrary to the entropy. Therefore, the entropy term can be omitted for the continuous flows when the entropy can be considered as a continuous function depending on the Lagrange markers.

From the velocity representation Eq. (16) and the equations of motion (4) - (11) it strictly follows that the velocity field $\mathbf{v}=\mathbf{v}_{0}$ satisfies Euler equation with the magnetic force taken into account. Namely, providing differentiation we have

$$
\rho D \mathbf{v}_{0}=-\nabla p+\frac{\operatorname{curl} \mathbf{H} \times \mathbf{H}}{4 \pi}
$$

where $p$ is the fluid pressure. 


\section{A. Canonical variables.}

The variational principle can be easily reformulated in the Hamiltonian form. Excluding the magnetic and velocity fields by means of Eqs. (12), (16) we arrive at the following Hamiltonian density

$$
\mathcal{H}=\mathcal{H}(\mathcal{P}, \nabla \mathcal{Q})=\mathcal{P} \partial_{t} \mathcal{Q}-\mathcal{L}^{\prime}=\rho \frac{v_{0}^{2}}{2}+\rho \varepsilon(\rho, s)+\frac{(\operatorname{curl} \mathbf{A})^{2}}{8 \pi}-\mathbf{M} \cdot \nabla \Lambda .
$$

Equations of motion (4) - (11) can now be expressed in the canonical form

$$
\partial_{t} \mathcal{Q}=\delta \mathcal{H} / \delta \mathcal{P}, \quad \partial_{t} \mathcal{P}=-\delta \mathcal{H} / \delta \mathcal{Q}, \quad \mathcal{Q}=(\varphi, \boldsymbol{\mu}, s ; \mathbf{A}), \quad \mathcal{P}=(\rho, \boldsymbol{\lambda}, \sigma ;-\mathbf{M})
$$

Eq. (12) serves as a definition of the magnetic field, and the divergence-free condition for the subsidiary field $\mathbf{M}$, Eq. (13), follows from the variation of the action

$$
\mathcal{A}=\int d t \int d \mathbf{r}\left(\mathcal{P} \partial_{t} \mathcal{Q}-\mathcal{H}\right)
$$

with respect to $\Lambda$. Note that it is possible to put $\Lambda=0$. Under this assumption the divergence-free condition for the field $\mathbf{M}$ vanishes. But from Eq. (11) it follows that divM is a conserved quantity, $\partial_{t} \operatorname{div} \mathbf{M}=0$. Therefore, supposing that $\operatorname{div} \mathbf{M}=0$ holds for some initial moment we arrive at the conclusion that this is valid for the arbitrary moment. Nevertheless, it proves convenient to deal with $\Lambda \neq 0$ that makes it possible to use different gauge conditions for the vector potential.

The above variational principle results in the set of dynamic equations. From the latter follow the conventional MHD equations, (4), (9), (17) and the equation for the magnetic field, which follows from Eq. (10) after taking curl,

$$
\partial_{t} \mathbf{H}=\operatorname{curl}[\mathbf{v} \times \mathbf{H}] .
$$

On the contrary, if at some initial moment, $t=\bar{t}$, we have the conventional MHD fields $\bar{\rho}, \bar{s}$, $\overline{\mathbf{v}}$ and $\overline{\mathbf{H}}$, then we can find the initial subsidiary fields $\bar{\varphi}, \overline{\boldsymbol{\mu}}, \overline{\boldsymbol{\lambda}}, \bar{\sigma}, \overline{\mathbf{A}}, \overline{\mathbf{M}}$ and $\bar{\Lambda}$, satisfying Eqs. (12) - (14). This can be done to within the gauge transformations (the latter do not change both the velocity and the magnetic field) due to the fact that the subsidiary fields play a role of generalized potentials. Then, if the uniqueness conditions are satisfied both for the conventional MHD equations and for the set of variational equations, we are led to conclude that corresponding solutions coincide for all moments. In this sense we can state that these sets of equations are equivalent, cf. Ref. [4].

The complete representation of the velocity field in the form of the generalized Clebsch representation (16) allows, first, to deal with the MHD flows of general type, including all types of breaks, cf. Ref. 14]; second, for the zero magnetic field it results in the correct limit transition to the conventional hydrodynamics, cf. Refs. [25, 26]; third, it allows obtaining the integrals and invariants of motion for the MHD flows additional to the known ones: for instance, the generalized Ertel invariant, generalized vorticity and generalized helicity, see below. The two last integrals were deduced for the particular case of incompressible flows in the papers [8, 9], cf. also papers [18, 19, 20] where the vorticity and helicity analogs were obtained for the MHD flows with the specific spatial symmetry.

Moreover, it is possible to show that representation (16) is equivalent to that following from the Weber transformation, cf. Refs. [23, 24] and the recent review [1].

\section{GENERALIZED WEBER TRANSFORMATION.}

Suppose that the fluid particles are labelled by Lagrange markers $\mathbf{a}=\left(a_{1}, a_{2}, a_{3}\right)$. The label of the particle passing through point $\mathbf{r}=\left(x_{1}, x_{2}, x_{3}\right)$ at time $t$ is then

$$
\mathbf{a}=\mathbf{a}(\mathbf{r}, t), \quad D \mathbf{a}=\frac{\partial \mathbf{a}}{\partial t}+(\mathbf{v} \cdot \nabla) \mathbf{a}=0
$$

The particle paths and velocities are given by the inverse function

$$
\mathbf{r}=\mathbf{r}(\mathbf{a}, t), \quad \mathbf{v}=D \mathbf{r}(\mathbf{a}, t)=\partial \mathbf{r} /\left.\partial t\right|_{\mathbf{a}=\text { const }} .
$$

Let the initial position of the particle labelled $\mathbf{a}$ is $\mathbf{X}$, i.e.,

$$
\mathbf{r}(\mathbf{a}, 0)=\mathbf{X}(\mathbf{a})
$$


A natural choice of the labels would be $\mathbf{X}(\mathbf{a})=\mathbf{a}$; however it is convenient to retain the extra freedom represented by the "rearrangement function" $\mathbf{X}(\mathbf{a})$.

We seek to transform the equation of motion (17) to an integrable form, by generalizing the argument of Weber [23. (see, for example, Refs. [29], [1], and [8]. It is convenient to represent Eq. (17) as

$$
D \mathbf{v}=-\nabla w+T \nabla s+\mathbf{j} \times \mathbf{h},
$$

where $\mathbf{h}=\mathbf{H} / \rho$ and the vector $\mathbf{j}$ is defined according to

$$
\mathbf{j}=\frac{\operatorname{curl} \mathbf{H}}{4 \pi}
$$

being proportional to the current density. Multiplying Eq. (25) by $\partial x_{k} / \partial a_{i}$ we have

$$
\left(D v_{k}\right) \frac{\partial x_{k}}{\partial a_{i}}=-\frac{\partial w}{\partial x_{k}} \frac{\partial x_{k}}{\partial a_{i}}+T \frac{\partial s}{\partial x_{k}} \frac{\partial x_{k}}{\partial a_{i}}+[\mathbf{j} \times \mathbf{h}]_{k} \frac{\partial x_{k}}{\partial a_{i}} .
$$

The 1.h.s. can be represented as

$$
\left(D v_{k}\right) \frac{\partial x_{k}}{\partial a_{i}}=D\left(v_{k} \frac{\partial x_{k}}{\partial a_{i}}\right)-\frac{\partial}{\partial a_{i}}\left(v^{2} / 2\right),
$$

where we have taken into account that operator $D \equiv \partial /\left.\partial t\right|_{\mathbf{a}=\text { const }}$ and therefore $D x_{k}=v_{k}$ and $D$ commutes with derivative $\partial / \partial a_{i}$. Eq. (27) now takes the form

$$
D\left(v_{k} \frac{\partial x_{k}}{\partial a_{i}}\right)=\frac{\partial}{\partial a_{i}}\left(v^{2} / 2-w\right)+T \frac{\partial s}{\partial a_{i}}+[\mathbf{j} \times \mathbf{h}]_{k} \frac{\partial x_{k}}{\partial a_{i}}
$$

It is convenient to transform the last term by means of the dynamic equation for the subsidiary field $\mathbf{m}=\mathbf{M} / \rho$ (compare Eq. (11))

$$
D \mathbf{m}=(\mathbf{m} \cdot \nabla) \mathbf{v}+\mathbf{j} / \rho .
$$

Then we can transform the last term in the r.h.s. of Eq. 29) to the form of the substantial derivative, see Appendix,

$$
[\mathbf{j} \times \mathbf{h}]_{k} \frac{\partial x_{k}}{\partial a_{i}}=D\left([\mathbf{m} \times \mathbf{H}]_{k} \frac{\partial x_{k}}{\partial a_{i}}\right) .
$$

Analogously, the first two terms in the r.h.s. of Eq. 29) can be presented as substantial derivatives by means of introducing subsidiary functions $\varphi$ and $\sigma$, which satisfy equations (compare Eqs. (9), (5))

$$
\begin{gathered}
D\left(\frac{\sigma}{\rho}\right)=-T, \\
D \varphi=w-v^{2} / 2 .
\end{gathered}
$$

Then

$$
T \frac{\partial s}{\partial a_{i}}=-\frac{\partial s}{\partial a_{i}} D\left(\frac{\sigma}{\rho}\right)=-D\left(\frac{\partial s}{\partial a_{i}} \frac{\sigma}{\rho}\right), \quad \frac{\partial}{\partial a_{i}}\left(v^{2} / 2-w\right)=-D\left(\frac{\partial \varphi}{\partial a_{i}}\right)
$$

where we have taken into account that $D s=0$ along with $D\left(\partial s / \partial a_{i}\right)=0$. Therefore, we can present the Euler equation (29) in the integrable form

$$
D\left(v_{k} \frac{\partial x_{k}}{\partial a_{i}}\right)=-D\left(\frac{\partial \varphi}{\partial a_{i}}\right)-D\left(\frac{\partial s}{\partial a_{i}} \frac{\sigma}{\rho}\right)+D\left([\mathbf{m} \times \mathbf{H}]_{k} \frac{\partial x_{k}}{\partial a_{i}}\right) .
$$

Integration leads to the relation

$$
v_{k} \frac{\partial x_{k}}{\partial a_{i}}=-\frac{\partial \varphi}{\partial a_{i}}-\frac{\partial s}{\partial a_{i}} \frac{\sigma}{\rho}-[\mathbf{H} \times \mathbf{m}]_{k} \frac{\partial x_{k}}{\partial a_{i}}+b_{i}
$$


Here $\mathbf{b}=\mathbf{b}(\mathbf{a})$ does not depend on time explicitly, $D \mathbf{b}=0$, presenting the vector constant of integration. Multiplying this relation by $\partial a_{i} / \partial x_{j}$ allows reverting from Lagrangian $(\mathbf{a}, t)$, to the Eulerian, $(\mathbf{r}, t)$, variables,

$$
\mathbf{v}=-\nabla \varphi+b_{k} \nabla a_{k}-\frac{\sigma}{\rho} \nabla s-\mathbf{h} \times \mathbf{M} .
$$

This representation obviously coincides with the above discussed Clebsch representation if one identifies $\mathbf{b}$ with $-\boldsymbol{\lambda} / \rho$ and $\mathbf{a}$ with $\boldsymbol{\mu}$. Moreover, this proves equivalence of description of the general-type magnetohydrodynamic flows in terms of canonical variables introduced and the conventional description in Lagrange or Euler variables. The equations of motion for the generalized coordinates and momenta follow now from definitions of the subsidiary variables $\mathbf{a}, \mathbf{m}=\mathbf{M} / \rho, \sigma, \varphi$ and $\mathbf{b}$.

Emphasize that the vector field $\mathbf{M}=\rho \mathbf{m}$ introduced by Eq. (30) satisfies the integral relation

$$
\partial_{t} \int_{\Sigma} \mathbf{M} \cdot d \boldsymbol{\Sigma}=\int_{\Sigma} \mathbf{j} \cdot d \mathbf{\Sigma}
$$

where $\boldsymbol{\Sigma}$ is some oriented area moving with the fluid. This fact was first indicated in Ref. [8] for the incompressible flows. Now we see that it holds true for the general case. The proof of this statement is given in Appendix. Expressing $\mathbf{M}=$ curlS and making use of the Stokes theorem we conclude that time derivative of the vector $\mathbf{S}$ circulation over the closed frozen-in contour $\partial \Sigma$ is proportional to the current (recall, $\mathbf{j}=(4 \pi)^{-1}$ curlH and differs from the current density by the constant multiplier) intersecting the surface defined by this contour,

$$
\partial_{t} \int_{\partial \Sigma} \mathbf{S} \cdot d \mathbf{l}=\int_{\Sigma} \mathbf{j} \cdot d \mathbf{\Sigma}=(4 \pi)^{-1} \int_{\partial \Sigma} \mathbf{H} \cdot d \mathbf{l}
$$

that highlights the physical meaning of the subsidiary field $\mathbf{S}$ usually introduced for the canonical description of MHD flows. Underline that this identity strictly follows from the dynamic equation for the subsidiary field $\mathbf{S}$ and is insensitive to the compressibility.

The vector constant of integration, $\mathbf{b}$, may be expressed in terms of the initial conditions,

$$
\begin{gathered}
b_{i}=\bar{V}_{k}(\mathbf{a}) \frac{\partial X_{k}}{\partial a_{i}}+\frac{\partial \varphi_{0}}{\partial a_{i}}+c_{0} \frac{\partial s}{\partial a_{i}}, \\
\varphi_{0}=\varphi(\mathbf{a}, 0), \quad c_{0}=\left.\left(\frac{\sigma}{\rho}\right)\right|_{t=0}, \quad \bar{V}_{k}(\mathbf{a})=V_{k}(\mathbf{a})+\left[\mathbf{h}_{0} \times \mathbf{M}_{0}\right]_{k}, \quad V_{k}(\mathbf{a})=v_{k}(\mathbf{a}, 0), \\
\mathbf{h}_{0} \equiv \mathbf{h}_{0}(\mathbf{a})=\mathbf{h}(\mathbf{x}(\mathbf{a}, 0), 0)=\mathbf{h}(\mathbf{X}(\mathbf{a}), 0), \quad \mathbf{M}_{0} \equiv \mathbf{M}_{0}(\mathbf{a})=\mathbf{M}(\mathbf{x}(\mathbf{a}, 0), 0)=\mathbf{M}(\mathbf{X}(\mathbf{a}), 0) .
\end{gathered}
$$

Under special conditions, namely, for

$$
\mathbf{X}(\mathbf{a})=\mathbf{a}, \quad \mathbf{r}(\mathbf{a}, 0)=\mathbf{a}, \quad \mathbf{a}(\mathbf{r}, 0)=\mathbf{r},
$$

from Eq. (40) it follows

$$
b_{i}=\bar{V}_{i}(\mathbf{a})+\frac{\partial \varphi_{0}}{\partial a_{i}}+c_{0} \frac{\partial s}{\partial a_{i}} .
$$

Adopting zero initial conditions,

$$
\mathbf{M}_{0}=0, \quad \varphi_{0}=0, \quad \sigma_{0}=0,
$$

we obtain

$$
\mathbf{b}=\overline{\mathbf{V}}(\mathbf{a})=\widetilde{\mathbf{v}}(\mathbf{a}, 0) \equiv \widetilde{\mathbf{v}}_{0}(\mathbf{a})=\mathbf{v}(\mathbf{a}, 0),
$$

where symbol $\sim$ indicates that we are dealing with the velocity field in the Lagrange description, i.e., $\widetilde{\mathbf{v}}(\mathbf{a}, t)$ denotes the velocity of the fluid particle with label $\mathbf{a}$ at time $t$. Evidently, $\widetilde{\mathbf{v}}(\mathbf{a}, t)=\mathbf{v}(\mathbf{r}, t)$, where $\mathbf{a}$ and $\mathbf{r}$ are linked by relations (22) and (23) for the specific choice given by Eqs. (41), (43). Then the velocity representation takes the particular form

$$
\mathbf{v}=\mathbf{v}_{h}-[\mathbf{h} \times \mathbf{M}], \quad \mathbf{v}_{h} \equiv-\nabla \varphi+\widetilde{v}_{0 k} \nabla a_{k}-\frac{\sigma}{\rho} \nabla s, \quad \widetilde{\mathbf{v}}_{0}(\mathbf{r})=\mathbf{v}(\mathbf{r}, 0), \quad \mathbf{a}(\mathbf{r}, 0)=\mathbf{r} .
$$

It differs from that presented in Ref. [4] by involving the entropy term. Note that existence of this term allows one to describe the general-type MHD flows (and hydrodynamic flows under condition $\mathbf{H}=0$ ) with arbitrary possible discontinuities, including shocks, slides and rotational breaks, cf. Ref. 14, 25, 26]. One can omit this term for continuous barotropic and isentropic flows. 


\section{INTEGRALS OF MOTION.}

The conservation laws, as is well-known, follow from the specific symmetries of the action. Existence of the relabelling transformations group (first discussed by Salmon in Ref. 30]) of the Lagrange markers, $\boldsymbol{\mu}$, leads to the integrals of motion that are additional to the energy, the fluid momentum and mass conservation. These additional integrals are expressed in terms of the Lagrange description of the motion, i.e., in terms of the Lagrange markers, etc. Therefore, as a rule, they are gauge-dependent. The frozen-in character of the magnetic field results in the specific topological integrals of motion, namely, magnetic helicity and cross-helicity, first discussed in Refs. 15, 16, 17], see also review [1]. Corresponding densities are respectively

$$
h_{M}=\mathbf{A} \cdot \mathbf{H},
$$

and

$$
h_{C}=\mathbf{v} \cdot \mathbf{H} .
$$

To clarify the following discussion relating to the additional local invariants and integrals of motion, let us briefly recall the known ones. As it strictly follows from the dynamic equations, the local conservation law for the magnetic helicity holds true for general type MHD flows

$$
\partial_{t} h_{M}+\operatorname{div} \mathbf{q}_{M}=0, \quad \mathbf{q}_{M}=\mathbf{v} h_{M}-\mathbf{H} \cdot(\mathbf{A} \cdot \mathbf{v}-\Lambda) .
$$

On the contrary, in the general case the cross-helicity is governed by equation

$$
\partial_{t} h_{C} / \partial t=-\operatorname{div}\left[\mathbf{v} h_{C}+\left(w-v^{2} / 2\right) \mathbf{H}\right]+T \operatorname{div}(s \mathbf{H})
$$

and is not conserved. But for barotropic and isentropic flows the pressure $p=p(\rho)$ and $h_{C}$ is conserved,

$$
\partial_{t} h_{C}+\operatorname{divq}_{C}=0, \quad \mathbf{q}_{C}=\mathbf{v} h_{C}+\left(\chi-v^{2} / 2\right) \mathbf{H},
$$

where $\chi=\int d p / \rho$.

For the general case one more conserved quantity first discovered by Gordin and Petviashvili, cf. Ref. [31], is known. Corresponding density is

$$
h_{P}=\mathbf{H} \cdot \nabla s
$$

and

$$
\partial_{t} h_{P}+\operatorname{div} \mathbf{q}_{P}=0, \quad \mathbf{q}_{P}=\mathbf{v} h_{P} .
$$

The integral conservation laws are related to the local conserved quantities. For instance, integrating $h_{P}$ over arbitrary substantial volume $\widetilde{V}$ we obtain conserved quantity $\mathcal{I}_{P}$,

$$
\mathcal{I}_{P}=\int_{\widetilde{V}} d \mathbf{r} h_{P}, \quad \partial_{t} \mathcal{I}_{P}=0
$$

Note that $h_{P} / \rho$ gives us an example of the so-called local Lagrange invariants (in other words, Casimirs), cf. Refs. [10, 11, 12, 13] and 1, 2]. By definition they obey the following equations

$$
\begin{gathered}
\partial_{t} \alpha+\mathbf{v} \cdot \nabla \alpha=0, \quad \partial_{t} \mathbf{I}+(\mathbf{v} \cdot \nabla) \mathbf{I}=0, \\
\partial_{t} \mathbf{J}+(\mathbf{v} \cdot \nabla) \mathbf{J}-(\mathbf{J} \cdot \nabla) \mathbf{v}=0,
\end{gathered}
$$

Here $\alpha$ and $\mathbf{I}$ denote the scalar and vector Lagrange invariants, $\mathbf{J}$ is the frozen-in field, and $\mathbf{L}$ denotes $S$-type invariant by terminology of Ref. [12], related to a frozen-in surface. To these invariants it is necessary to add the density $\rho$. [36] 
Evidently, the quantity $h_{P} / \rho$ is the $\alpha$-type invariant. The Lagrange markers $\boldsymbol{\mu}$ and quantities $\boldsymbol{\lambda} / \rho$ supply examples of the vector Lagrange invariants, the magnetic field $\mathbf{H}$ divided by $\rho, \mathbf{h}=\mathbf{H} / \rho$, is invariant of the $\mathbf{J}$ - type, gradient of any scalar Lagrange invariant is the $S$-type invariant,

$$
\mathbf{L}^{\prime}=\nabla \alpha .
$$

There also exist other relations between different type invariants, see Refs. [1, 2], allowing one to generate new invariants. For instance, the scalar product of the $\mathbf{J}$ and $\mathbf{L}$ invariants results in some scalar Lagrange invariant, symbolically

$$
\alpha^{\prime}=(\mathbf{J} \cdot \mathbf{L})
$$

The above mentioned invariant $h_{P} / \rho$ can be obtained by means of this relation if we put $\mathbf{J}=\mathbf{h}$ and $\mathbf{L}=\nabla s$. Other examples are represented by relations generating $\mathbf{J}-(\mathbf{L})$ - type invariants by means of two L- $\left(\mathbf{J}_{-}\right)$type invariants,

$$
\begin{gathered}
\mathbf{J}^{\prime}=\left[\mathbf{L} \times \mathbf{L}^{\prime}\right] / \rho, \\
\mathbf{L}^{\prime}=\rho\left[\mathbf{J} \times \mathbf{J}^{\prime}\right] .
\end{gathered}
$$

Note that integrating of the density $h_{M}$ over an arbitrary substantial volume does not lead to the conserved integral. It is easy to check up that

$$
\mathcal{I}_{M}=\int_{\widetilde{V}} d \mathbf{r} h_{M}
$$

satisfies

$$
\partial_{t} \mathcal{I}_{M}=\int_{\partial \widetilde{V}} d \Sigma(\mathbf{A} \cdot \mathbf{v}-\Lambda) H_{n}, \quad H_{n}=\mathbf{H} \cdot \mathbf{n}
$$

where integration in the r.h.s. is performed over the boundary $\partial \widetilde{V}$ of the volume $\widetilde{V}, \mathbf{n}$ is the outward normal and $d \Sigma$ denotes an infinitesimal area of the surface $\partial \widetilde{V}$. It is obvious that $\mathcal{I}_{M}$ will be an integral of motion if $H_{n}$ equals zero. This fact allows us to conclude that $\mathcal{I}_{M}$ becomes an integral of motion if we choose the substantial volume in such a way that on the boundary of the initial volume, $\left.\widetilde{V}\right|_{t=t_{0}}$, holds equality $\left.H_{n}\right|_{t=t_{0}}=0$. The latter condition is invariant of the motion: if equality $H_{n}=0$ is fulfilled for the initial moment, then it holds true in the future.

Another way to make $\mathcal{I}_{M}$ invariant consists in fixing the gauge of the vector potential $\mathbf{A}$ so that $\mathbf{A} \cdot \mathbf{v}=\Lambda$. Then the dynamic equation for $\mathbf{A}$, (10), takes the form

$$
\partial_{t} \mathbf{A}+\nabla(\mathbf{v} \cdot \mathbf{A})-\mathbf{v} \times \operatorname{curl} \mathbf{A}=0
$$

i.e., A becomes an invariant of the $\mathbf{L}$ - type. Under this gauge condition the quantity $h_{M} / \rho$ presents the scalar Lagrange invariant, $D\left(h_{M} / \rho\right)=0$.

As for the local conservation law for the cross-helicity, Eq. [49), it obviously leads to the integral conserved quantity $\mathcal{I}_{C}$ for the barotropic flows but with the following restriction: integration has to be performed over the specific substantial volume, namely such that condition $\left.H_{n}\right|_{\partial \widetilde{V}}=0$ (this condition is invariant of the motion) holds,

$$
\partial_{t} \mathcal{I}_{C}=0, \quad \mathcal{I}_{C} \equiv \int_{\widetilde{V}} d \mathbf{r} h_{C},\left.\quad H_{n}\right|_{\partial \widetilde{V}}=0 .
$$

Existence of the recursive procedure allowing one to construct new invariants on the basis of the starting set of invariants, see Refs. 1, 2], underlines the role of the local invariants among other conserved quantities. Although in terms of the Lagrangian variables (such as the markers $\boldsymbol{\mu}$ ) there exists a wide set of invariants, see, for instance, Ref. [1], the most interesting invariants are such that can be expressed in Eulerian (physical) variables and thus are gauge-invariant.

Emphasize that in the conventional hydrodynamics there exists Ertel invariant $\alpha_{E}$,

$$
\alpha_{E}=h_{E} / \rho, \quad h_{E}=\omega \cdot \nabla s,
$$

where $\boldsymbol{\omega}=$ curlv is vorticity,

$$
\partial_{t} h_{E}+\operatorname{div} \mathbf{q}_{E}=0, \quad \mathbf{q}_{E}=h_{E} \mathbf{v}, \quad D \alpha_{E}=0 .
$$


The corresponding integral of motion reads

$$
\partial_{t} \mathcal{I}_{E}=0, \quad \mathcal{I}_{E} \equiv \int_{\widetilde{V}} d \mathbf{r} h_{E}
$$

Note that $D \mathcal{I}_{E}=0$ holds true for an arbitrary substantial volume $\widetilde{V}$.

The Ertel invariant density has the structure of the Eq. (57) with $\mathbf{L}=\nabla s, \mathbf{J}=\boldsymbol{\omega} / \rho$ (recall that $\boldsymbol{\omega}$ is a frozen-in field for the barotropic hydrodynamic flows). In the hydrodynamic case there also exists the helicity invariant

$$
h_{H}=\boldsymbol{\omega} \cdot \mathbf{v}
$$

which has a topological meaning, defining knottedness of the flow. It satisfies equation

$$
\partial_{t} h_{H}+\operatorname{divq}_{H}=0, \quad \mathbf{q}_{H}=h_{H} \mathbf{v}+\left(\chi-v^{2} / 2\right) \boldsymbol{\omega},
$$

and evidently results in the corresponding integral conservation law

$$
\partial_{t} \mathcal{I}_{H}=0, \quad \text { for }\left.\quad \omega_{n}\right|_{\partial \widetilde{V}}=0, \quad \mathcal{I}_{H} \equiv \int_{\widetilde{V}} d \mathbf{r} h_{H} .
$$

For the MHD case the vector $\boldsymbol{\omega} / \rho$ is not the frozen-in field due to the fact that magnetic force is non-potential. It seems evident that for the MHD case there have to exist the integrals of motion generalizing the conventional helicity and Ertel invariant along with vorticity integral. These invariants are to pass into the conventional ones for the vanishing magnetic field. The generalization for the vorticity and helicity invariants was obtained in the paper [8] for the particular case of the incompressible flows. In the following section it is shown that there exists MHD generalization for the Ertel invariant, and results of the paper [8] relating to the vorticity and helicity can be extended to incompressible barotropic MHD flows.

\section{A. Generalized vorticity.}

Let us prove that the quantity $\boldsymbol{\omega}_{h} / \rho$, where

$$
\boldsymbol{\omega}_{h} \equiv \operatorname{curl}_{h}=-\left[\nabla\left(\frac{P}{\rho}\right) \times \nabla Q\right]=-\left[\nabla\left(\frac{\lambda_{m}}{\rho}\right) \times \nabla \mu_{m}\right]-\left[\nabla\left(\frac{\sigma}{\rho}\right) \times \nabla s\right],
$$

is the frozen-in field ("hydrodynamic" part of the vorticity) for the barotropic MHD flows. It would be a trivial consequence of the fact that $\left[\mathbf{L} \times \mathbf{L}^{\prime}\right] / \rho$, where $\mathbf{L}, \mathbf{L}^{\prime}$ are Lamb type invariants, is the local invariant of the frozen-in type if all quantities $Q$ and $P / \rho$ satisfy homogeneous transport equations being $\alpha$ - or $\mathbf{I}$ - type invariants (remember that $\nabla \alpha$ and $\nabla I_{m}$ are $\mathbf{L}$-type invariants). But $\varphi$ and $\sigma / \rho$ satisfy the inhomogeneous equations of motion. Therefore, let us start with equation of motion for the "hydrodynamic" part of the velocity. Differentiating (16) and making use of relations

$$
D(\nabla X)=\nabla(D X)-\left(\nabla v_{m}\right) \cdot \partial_{m} X
$$

we have

$$
D \mathbf{v}_{h}=-D\left(\frac{P}{\rho}\right) \cdot \nabla Q-\frac{P}{\rho} \cdot \nabla(D Q)+\frac{P}{\rho}\left(\nabla v_{m}\right) \cdot \partial_{m} Q=T \nabla s-\nabla\left(w-v^{2} / 2\right)-v_{h m} \nabla v_{m},
$$

or, after simple rearrangements,

$$
D \mathbf{v}_{h}=-\nabla p / \rho+\left(v_{m}-v_{h m}\right) \cdot \nabla v_{m} .
$$

Taking the curl of this equation results in

$$
\begin{aligned}
\partial_{t} \boldsymbol{\omega}_{h}= & -\operatorname{curl}\left(v_{m} \partial_{m} \mathbf{v}_{h}\right)+[\nabla \rho \times \nabla p] / \rho^{2}-\operatorname{curl}\left(v_{h m} \nabla v_{m}\right)= \\
& =[\nabla \rho \times \nabla p] / \rho^{2}+\operatorname{curl}\left(v_{m} \nabla v_{h m}-v_{h m} \nabla v_{m}\right) .
\end{aligned}
$$


The term in the square brackets is equal to $v_{m} \nabla v_{h m}-v_{h m} \nabla v_{m}=\mathbf{v} \times \boldsymbol{\omega}_{h}$ and we obtain

$$
\partial_{t} \boldsymbol{\omega}_{h}=[\nabla \rho \times \nabla p] / \rho^{2}+\operatorname{curl}\left[\mathbf{v} \times \boldsymbol{\omega}_{h}\right] .
$$

For the barotropic flows, $p=p(\rho)$, the first term in the r.h.s. becomes zero and we can see that $\boldsymbol{\omega}_{h} / \rho$ is the frozen-in field,

$$
D\left(\frac{\boldsymbol{\omega}_{h}}{\rho}\right)=\left(\frac{\boldsymbol{\omega}_{h}}{\rho} \cdot \nabla\right) \mathbf{v}
$$

At $\mathbf{H}=0, \boldsymbol{\omega}_{h}$ corresponds to the conventional hydrodynamic vorticity.

\section{B. Generalized Kelvin's theorem.}

The frozen-in character of the generalized vorticity allows obtaining the strict generalization of the Kelvin's theorem for the barotropic flows. But with some restrictions it is valid also for the non barotropic flows. Namely, circulation $\Gamma$ of the hydrodynamic part of the velocity over the closed material contour $\mathcal{C}$ is a constant of motion if the entropy $s$ is constant on this contour,

$$
D \Gamma=0, \quad \Gamma \equiv \oint_{\mathcal{C}} \mathbf{v}_{h} \cdot d \mathbf{l} \quad \text { for }\left.\quad s\right|_{C}=\text { const }
$$

The proof strictly follows from the velocity representation:

$$
\Gamma=\oint_{\mathcal{C}} \mathbf{v}_{h} \cdot d \mathbf{l}=\oint_{\mathcal{C}}\left(d \varphi+\frac{\lambda_{m}}{\rho} d \mu_{m}+\frac{\sigma}{\rho} d s\right)=\oint_{\mathcal{C}}\left(\frac{\lambda_{m}}{\rho} d \mu_{m}+\frac{\sigma}{\rho} d s\right) .
$$

Differentiating $\Gamma$ and taking into account that $D \mu_{m}=D\left(\lambda_{m} / \rho\right)=0$ we obtain

$$
D \Gamma=\oint_{\mathcal{C}} d s D\left(\frac{\sigma}{\rho}\right)=-\oint_{\mathcal{C}} T d s=0 \quad \text { for }\left.\quad s\right|_{\mathcal{C}}=\text { const } .
$$

Note that for barotropic flows this result strictly follows from the fact that $\boldsymbol{\omega}_{h} / \rho$ is the frozen-in field. Namely, for any $\mathbf{J}$-type invariant it can be easily proved that

$$
D \int_{\Sigma} d \Sigma \rho \mathbf{J} \cdot \mathbf{n}=0
$$

where integration is performed over the substantial surface $\Sigma$. Then for $\rho \mathbf{J}=\boldsymbol{\omega}_{h}$ after applying the Stokes theorem, we have

$$
D \int_{\Sigma} d \Sigma \boldsymbol{\omega}_{h} \cdot \mathbf{n}=D \oint_{\partial \Sigma} \mathbf{v}_{h} \cdot d \mathbf{l}=0
$$

\section{Generalized helicity.}

Now it can be proved that generalized helicity, $h_{H}$, defined in terms of the "hydrodynamic" part of the velocity,

$$
h_{H}=\omega_{h} \cdot \mathbf{v}_{h},
$$

is the integral of motion for barotropic flows. Differentiating Eq. (75) and taking into account Eqs. (69), (70) for barotropic flows we arrive at the local conservation law of the form (rather cumbersome calculations are given in Appendix):

$$
\partial_{t} h_{H}+\operatorname{divq}_{H}=0, \quad \mathbf{q}_{H}=h_{H} \mathbf{v}+\left(\chi-v^{2} / 2\right) \boldsymbol{\omega}_{h}
$$

In analogy with the hydrodynamic case we can conclude that the integral helicity $\mathcal{I}_{H}$ (defined by means of Eq. (67)) is the integral invariant, moving together with the fluid if the normal component of the vorticity tends to zero, $\omega_{h n}=0$, on the surface of the corresponding substantial volume $\widetilde{V}$. Note that the condition $\omega_{h n}=0$ is invariant of the motion (due to the frozen-in character of $\boldsymbol{\omega}_{h} / \rho$ ) and therefore it can be related to the initial surface only. 


\section{Generalized Ertel invariant.}

Let us show that there exists strict generalization of the Ertel invariant for the MHD case. For this purpose let us prove that without any restrictions related to the character of the flow the quantity

$$
h_{E}=\left(\boldsymbol{\omega}_{h} \cdot \nabla s\right)
$$

obeys the conservation law of the form

$$
\partial_{t} h_{E}+\operatorname{div} \mathbf{q}_{E}=0, \quad \mathbf{q}_{E}=h_{E} \mathbf{v}
$$

Equivalently, the quantity $\alpha_{E}=h_{E} / \rho$ is transported by the fluid

$$
D \alpha_{E}=0, \quad \alpha_{E}=h_{E} / \rho,
$$

being $\alpha$-type invariant. For the barotropic flows it immediately follows from the fact that $\boldsymbol{\omega}_{h} / \rho$ is the frozen-in field if the composition rules given by Eqs. (57) and (56) are taken into account. In order to make the proof for the non barotropic flows more transparent let us consider a more general situation. Let $\widetilde{\mathbf{J}}$ satisfy equation of motion of the form

$$
D \widetilde{\mathbf{J}}=(\widetilde{\mathbf{J}} \cdot \nabla) \mathbf{v}+\mathbf{Z}
$$

differing from the frozen field equation (154) by existence of the term $\mathbf{Z}$ that violates homogeneity. Then, if $\alpha$ represents any scalar Lagrange invariant, we have

$$
D(\widetilde{\mathbf{J}} \cdot \nabla \alpha)=D \widetilde{\mathbf{J}} \cdot \nabla \alpha+\widetilde{\mathbf{J}} \cdot D \nabla \alpha=\mathbf{Z} \cdot \nabla \alpha+((\widetilde{\mathbf{J}} \cdot \nabla) \mathbf{v}) \cdot \nabla \alpha-\left(\widetilde{\mathbf{J}} \cdot \nabla v_{m}\right) \cdot \partial_{m} \alpha
$$

Here the two last terms cancel and we get

$$
D(\widetilde{\mathbf{J}} \cdot \nabla \alpha)=\mathbf{Z} \cdot \nabla \alpha \quad \text { if } \quad D \widetilde{\mathbf{J}}=(\widetilde{\mathbf{J}} \cdot \nabla) \mathbf{v}+\mathbf{Z} \quad \text { and } \quad D \alpha=0
$$

For $\mathbf{Z}=0$ these relations prove the generating rule of Eq. (57). But we can see that $\widetilde{\mathbf{J}} \cdot \nabla \alpha$ becomes the local Lagrange invariant under less restrictive condition $\mathbf{Z} \cdot \nabla \alpha=0$. That is the case for the Ertel invariant: $\mathbf{Z}=[\nabla \rho \times \nabla p] / \rho^{3}$ is orthogonal to $\nabla s$ due to the fact that the scalar product of any three thermodynamic quantities is equal to zero (because any thermodynamic variable in the equilibrium state is a function of two basic variables). This concludes the proof.

The conserved integral quantity associated with $\alpha_{E}$ is

$$
\mathcal{I}_{E}=\int_{\widetilde{V}} d \mathbf{r} h_{E}, \quad \partial_{t} \mathcal{I}_{E}=0
$$

Note that by the structure $\mathcal{I}_{E}$ is not gauge-invariant in contrast to the hydrodynamic case. Let us examine its change under gauge transformation that results in $\mathbf{v}_{h} \Rightarrow \mathbf{v}_{h}^{\prime}, \mathbf{v}_{M} \Rightarrow \mathbf{v}_{M}^{\prime}$ with

$$
\mathbf{v}_{h}^{\prime}+\mathbf{v}_{M}^{\prime}=\mathbf{v}_{h}+\mathbf{v}_{M}
$$

Then

$$
\mathcal{I}_{E}^{\prime}-\mathcal{I}_{E}=\int_{\widetilde{V}} d \mathbf{r} \nabla s \cdot\left(\boldsymbol{\omega}_{h}^{\prime}-\boldsymbol{\omega}_{h}\right)=\int_{\widetilde{V}} d \mathbf{r} \nabla s \cdot\left(\boldsymbol{\omega}_{M}-\boldsymbol{\omega}_{M}^{\prime}\right) .
$$

But $\nabla s \cdot\left(\boldsymbol{\omega}_{M}-\boldsymbol{\omega}_{M}^{\prime}\right)=-\operatorname{div}\left[\nabla s \times\left(\mathbf{v}_{M}^{\prime}-\mathbf{v}_{M}\right)\right]$ and, therefore,

$$
\mathcal{I}_{E}^{\prime}-\mathcal{I}_{E}=-\int_{\partial \widetilde{V}} d \Sigma \mathbf{n} \cdot\left[\nabla s \times\left(\mathbf{v}_{M}^{\prime}-\mathbf{v}_{M}\right)\right]
$$

Now we can proceed in the two ways. First, making use of identity $\nabla s \times \mathbf{X}=\operatorname{curl}(s \mathbf{X})-s \cdot \operatorname{curl} \mathbf{X}$ we obtain

$$
\mathcal{I}_{E}^{\prime}-\mathcal{I}_{E}=-\int_{\partial \widetilde{V}} d \Sigma \mathbf{n} \cdot\left(\operatorname{curl}\left(s\left(\mathbf{v}_{M}^{\prime}-\mathbf{v}_{M}\right)\right)-s \operatorname{curl}\left(\mathbf{v}_{M}^{\prime}-\mathbf{v}_{M}\right)\right)
$$


Here the integral of the first term vanishes (that is trivial for a closed boundary $\partial \widetilde{V}$ and assumes the necessary decrease of the integrand for the infinite volume $\widetilde{V}$ ) and we have

$$
\mathcal{I}_{E}^{\prime}-\mathcal{I}_{E}=\int_{\partial \widetilde{V}} d \Sigma s \mathbf{n} \cdot \operatorname{curl}\left(\mathbf{v}_{M}^{\prime}-\mathbf{v}_{M}\right) .
$$

This representation immediately suggests that integral Ertel invariant becomes gauge-independent for the substantial volume $\widetilde{V}$ chosen in such a way that its boundary coincides with the entropy-constant surface, $\left.s\right|_{\partial \widetilde{V}}=$ const.

The second way is as follows. Bearing in mind that $\mathbf{v}_{M}^{\prime}-\mathbf{v}_{M}=-\left[\mathbf{h} \times\left(\mathbf{M}^{\prime}-\mathbf{M}\right)\right]$ we obtain

$$
\mathcal{I}_{E}^{\prime}-\mathcal{I}_{E}=\int_{\partial \widetilde{V}} d \Sigma \mathbf{n} \cdot\left[\nabla s \times\left[\mathbf{h} \times\left(\mathbf{M}^{\prime}-\mathbf{M}\right)\right]\right] .
$$

Inasmuch as both $\mathbf{M}^{\prime}$ and $\mathbf{M}$ satisfy Eq. (11), their difference, $\overline{\mathbf{M}}=\mathbf{M}^{\prime}-\mathbf{M}$, is governed by the homogeneous equation

$$
\partial_{t} \overline{\mathbf{M}}=\operatorname{curl}[\mathbf{v} \times \overline{\mathbf{M}}],
$$

i.e. $\overline{\mathbf{m}}=\overline{\mathbf{M}} / \rho$ is frozen-in field. Then we can conclude that the vector $\nabla s \times[\mathbf{h} \times \overline{\mathbf{m}}]$ entering the integrand is the frozen-in field, as it follows from recursion relations (56) - (58). Therefore, if we adopt relation $\left.\mathbf{n} \cdot[\nabla s \times[\mathbf{h} \times \overline{\mathbf{m}}]]\right|_{\partial \widetilde{V}}=0$ as the initial condition, then it holds true for all moments. For instance, this relation is fulfilled if $H_{n}=0$ and $\bar{m}_{n}=0$ at the initial moment. Evidently, these two conditions cannot be fulfilled for an arbitrary gauge. But we can restrict ourselves to a such subset of the initial conditions for the subsidiary field $\mathbf{M}$ that $\left.\mathbf{M}\right|_{t=t_{0}}=\left.f \mathbf{H}\right|_{t=t_{0}}$, where $f$ is an arbitrary function. (Then $\operatorname{div} \mathbf{M}=\left.\operatorname{div} \mathbf{M}\right|_{t=t_{0}}=\left.\nabla f \cdot \mathbf{H}\right|_{t=t_{0}}$ is time-independent in accordance with equation (11), and for the particular choice of $f$ such that $\left.\mathbf{H}\right|_{t=t_{0}} \cdot \nabla f=0$ we have $\operatorname{div} \mathbf{M}=0$.) For these initial conditions $\mathbf{M}$ along with $\mathbf{M}^{\prime}$ are collinear to $\mathbf{H}$ at the initial moment and therefore the initial value of the scalar product $\mathbf{n} \cdot[\nabla s \times[\mathbf{h} \overline{\mathbf{m}}]]$ is zero. Due to the frozen-in character of the quantity $\nabla s \times[\mathbf{h} \times \overline{\mathbf{m}}]$ equation $\mathbf{n} \cdot[\nabla s \times[\mathbf{h} \times \overline{\mathbf{m}}]]=0$ holds true for the arbitrary moment. Thus we can make the conclusion that gauge dependence of the Ertel's invariant can be partly eliminated by appropriate choice of the initial conditions or substantial volumes.

\section{E. Specific gauge.}

Examination of the integrals of motion shows that they are gauge-dependent. This dependence is attributed to different decompositions of the velocity field into the "hydrodynamic" and "magnetic" parts, $\mathbf{v}=\mathbf{v}_{h}+\mathbf{v}_{M}$. Underline that there exists a wide subgroup of the gauge transformations that include transformations that change the generalized potentials $\varphi, \boldsymbol{\mu}, \boldsymbol{\lambda}$ and $\sigma$ with no change in $\mathbf{M}$. The "hydrodynamic" part of the velocity representation, $\mathbf{v}_{h}$, does not evidently vary under the action of transformations of this, say, "hydrodynamic" subgroup. Then the generalized circulation, integral helicity and Ertel integral are likewise invariant under these gauge transformations. The simplest way to restrict the gauge transformations by this subgroup consists in adopting zero initial conditions for the subsidiary field M. This choice does not restrict in any way the character of a flow, in particular, all integrals of motion can possess nonzero values. The more detailed discussion of the gauge dependence of the additional integrals considered will be presented elsewhere.

\section{CONCLUSIONS.}

The results obtained can be summarized as follows. First, the variant of introducing the canonical description of the MHD flows by means of the variational principle with constraints is presented. It is shown that in order to describe general-type MHD flows it is necessary to use in the generalized Clebsch-type representation for the fluid velocity field the vector Clebsch variables (the Lagrange markers and conjugate momenta) along with the entropy term (cf. papers [25, 26] describing the hydrodynamic case) and the conventional magnetic term introduced first in Ref. [22]. Such a complete representation allows one to deal with general-type MHD flows, including all type of breaks, see Ref. 14. Second, it is proved that the generalized Weber transformation introduced leads to the velocity representation, which is equivalent to that introduced by means of the variational principle. Third, the existence of the generalized Ertel invariant for MHD flows is proven. Forth, there are generalized the vorticity and helicity invariants for the compressible barotropic MHD flows (first discussed for the incompressible case in cf. [8]). Fifth, the relations between the local and integral invariants are discussed along with the gauge dependence of the latter.

As a consequence of the completeness of the proposed velocity representation we get the correct limit transition from the MHD to the conventional hydrodynamic flows. The results obtained allow one to consider the complicated 
MHD problems in terms of the Hamiltonian variables. The use of this approach was demonstrated for the specific case of incompressible flows in the series of papers [8, 9] devoted to the nonlinear stability criteria. Emphasize that existence of the additional invariants proved in our paper is of high importance for the stability problems.

Note that existence of the additional basic invariants of the motion makes it actual to examine the problem of the complete set of independent invariants, and, respectively, the complete set of the corresponding Casimirs, cf. Ref. [1]. For instance, existence of the three independent basic local invariants for the non barotropic flows $\left(s, \alpha_{E}\right.$ and $\left.\mathbf{h}\right)$ immediately leads to the two denumerable sets of the monomial scalar invariants

$$
\alpha_{P}^{(m)}=(\mathbf{h} \cdot \nabla)^{m} s, \quad \alpha_{E}^{(m)}=(\mathbf{h} \cdot \nabla)^{m} \alpha_{E}, \quad \alpha_{P}^{(1)}=\alpha_{P}, \quad \alpha_{E}^{(0)}=\alpha_{E}, \quad m=0,1,2, \ldots
$$

The first set was discussed in the paper [1], and the second subset is a new one along with the "parent" Ertel invariant $\alpha_{E}$. Evidently,

$$
\widetilde{\alpha}=f\left(\left\{\alpha_{P}^{(m)}\right\},\left\{\alpha_{E}^{\left(m^{\prime}\right)}\right\}\right),
$$

where $f$ is an arbitrary function, is also the scalar Lagrange invariant. Therefore, we immediately arrive at the following set of integrals of motion (Casimirs)

$$
\mathcal{I}=\int_{\tilde{V}} d \mathbf{r} \rho f\left(\left\{\alpha_{P}^{(m)}\right\},\left\{\alpha_{E}^{\left(m^{\prime}\right)}\right\}\right)
$$

which is much wider than that discussed in the literature, cf. Eq. (10.23) in Ref. [1]. The additional set of the scalar monomial Lagrange invariants can be generated by the magnetic helicity under the specific gauge condition, $\Lambda=\mathbf{A} \cdot \mathbf{v}$, $\alpha_{M}^{(n)}=(\mathbf{h} \cdot \nabla)^{n} \alpha_{M}$. This enables evident generalization of the integrals of motion (85).

One example of the additional $\mathbf{J}$ - invariants reads

$$
\mathbf{J}^{\prime}=\left[\nabla s \times \nabla \alpha_{E}\right] / \rho .
$$

In turn, one can get new sets of the scalar invariants by applying operation $\left(\mathbf{J}^{\prime} \cdot \nabla\right)$ to the previous scalar invariants and so on. Obviously, this also leads to additional Casimirs to that indicated in (85).

For the barotropic flows the picture is analogous: the basic set of the scalar Lagrange invariants involves the generalized helicity, $\alpha_{H}=h_{H} / \rho$, and $\alpha_{M}$ (under the gauge condition specified above), and with $\mathbf{J}$-invariants $\mathbf{h}$ and $\boldsymbol{\omega}_{h}$. Therefore, we obtain additional scalar invariants $\alpha_{H}^{(n)}=(\mathbf{h} \cdot \nabla)^{n} \alpha_{H}, \alpha_{M}^{(n)}, \widetilde{\alpha}_{H}^{(n)}=\left(\rho^{-1} \boldsymbol{\omega}_{h} \cdot \nabla\right)^{n} \alpha_{H}, \widetilde{\alpha}_{M}^{(n)}=$ $\left(\rho^{-1} \boldsymbol{\omega}_{h} \cdot \nabla\right)^{n} \alpha_{M}$ and the conserved integrals of the form

$$
\mathcal{I}=\int_{\tilde{V}} d \mathbf{r} \rho f\left(\left\{\alpha_{H}^{(n)}\right\},\left\{\alpha_{M}^{\left(n^{\prime}\right)}\right\},\left\{\widetilde{\alpha}_{H}^{\left(n^{\prime \prime}\right)}\right\},\left\{\widetilde{\alpha}_{M}^{\left(n^{\prime \prime \prime}\right)}\right\}\right) .
$$

This set of the Casimirs generalizes that presented in cf. Ref. [1], the latter follows from (86) if we replace the function $f$ depending on the four sets of the monomial invariants by a function depending only on the invariants $\alpha_{M}^{(n)}$.

Note that we can construct the following generations of the local invariants by means of the recursion relations and obtain Casimirs of a more sophisticated structure than that presented in (85), (86). The problem of obtaining the complete set of the local invariants and gauge invariance of the corresponding integral invariants is rather complicated and is still under examination. This questions will be discussed in detail in the forthcoming paper.

\section{Appendix A}

In order to prove Eq. (31) let us substitute $\mathbf{j}$ from Eq. (30) into expression $[\mathbf{j} \times \mathbf{h}]_{k} \partial x_{k} / \partial a_{i}$. Then

$$
\begin{array}{r}
{[\mathbf{j} \times \mathbf{h}]_{k} \frac{\partial x_{k}}{\partial a_{i}}=[D \mathbf{m} \times \mathbf{H}]_{k} \frac{\partial x_{k}}{\partial a_{i}}-[(\mathbf{m} \cdot \nabla) \mathbf{v} \times \mathbf{H}]_{k} \frac{\partial x_{k}}{\partial a_{i}}=} \\
=\frac{\partial x_{k}}{\partial a_{i}} D\left([\mathbf{m} \times \mathbf{H}]_{k}\right)-\left([\mathbf{m} \times D(\rho \mathbf{h})]_{k}+[(\mathbf{m} \cdot \nabla) \mathbf{v} \times \mathbf{H}]_{k}\right) \frac{\partial x_{k}}{\partial a_{i}} .
\end{array}
$$

Proceeding with the terms in the second brackets we obtain

$$
\begin{array}{r}
{[\mathbf{m} \times D(\rho \mathbf{h})]_{k}+[(\mathbf{m} \cdot \nabla) \mathbf{v} \times \mathbf{H}]_{k}=[\mathbf{m} \times \mathbf{h}]_{k} \cdot D \rho+[\rho \mathbf{m} \times D \mathbf{h}]_{k}+[(\mathbf{m} \cdot \nabla) \mathbf{v} \times \mathbf{H}]_{k}=} \\
=-[\mathbf{M} \times \mathbf{h}]_{k} \cdot \operatorname{div} \mathbf{v}+[\mathbf{M} \times(\mathbf{h} \cdot \nabla) \mathbf{v}]_{k}+[(\mathbf{M} \cdot \nabla) \mathbf{v} \times \mathbf{h}]_{k}=-[\mathbf{M} \times \mathbf{h}]_{s} \partial_{k} v_{s},
\end{array}
$$


where $\mathbf{M}=\rho \mathbf{m}$ and the dynamic equation $D \mathbf{h}=(\mathbf{h} \cdot \nabla) \mathbf{v}$ along with identity

$$
[\mathbf{M} \times(\mathbf{h} \cdot \nabla) \mathbf{v}]_{k}+[(\mathbf{M} \cdot \nabla) \mathbf{v} \times \mathbf{h}]_{k}=[\mathbf{M} \times \mathbf{h}]_{k} \partial_{s} v_{s}-[\mathbf{M} \times \mathbf{h}]_{s} \partial_{k} v_{s}
$$

are taken into account. Introducing for brevity notation

$$
\mathbf{Y}=\mathbf{m} \times \mathbf{H} \equiv \mathbf{M} \times \mathbf{h},
$$

we can represent the r.h.s. of Eq. (87) as

$$
\frac{\partial x_{k}}{\partial a_{i}} \cdot D Y_{k}+Y_{s} \frac{\partial x_{k}}{\partial a_{i}} \partial_{k} v_{s}=\frac{\partial x_{k}}{\partial a_{i}} \cdot D Y_{k}+Y_{s} \frac{\partial v_{s}}{\partial a_{i}}=\frac{\partial x_{k}}{\partial a_{i}} \cdot D Y_{k}+Y_{s} \frac{\partial}{\partial a_{i}}\left(D x_{s}\right)=D\left(Y_{k} \frac{\partial x_{k}}{\partial a_{i}}\right) \text {. }
$$

This proves Eq. (31).

Let us check up the integral relation (38). It is sufficient to prove the differential form, namely

$$
D(\mathbf{M} \cdot d \boldsymbol{\Sigma})=\mathbf{j} \cdot d \boldsymbol{\Sigma},
$$

where $d \boldsymbol{\Sigma}$ is some infinitesimal oriented area moving with the fluid. It can be presented as

$$
d \boldsymbol{\Sigma}=d \mathbf{l}_{1} \times d \mathbf{l}_{2},
$$

where $d \mathbf{l}_{1}, d \mathbf{l}_{2}$ are frozen-in linear elements. Thus, $d \mathbf{l}_{a}, a=1,2$, are invariants of the $\mathbf{J}$ - type and satisfy equations

$$
D\left(d \mathbf{l}_{a}\right)=\left(d \mathbf{l}_{a} \cdot \nabla\right) \mathbf{v}
$$

Consequently, from the recursion relation Eq. (59) it follows that $\rho d \boldsymbol{\Sigma}$ is $\mathbf{L}$ - type invariant and hence it is governed by the dynamic equation

$$
D(\rho d \boldsymbol{\Sigma})=-\nabla(\rho \mathbf{v} \cdot d \boldsymbol{\Sigma})+\mathbf{v} \times \operatorname{curl}(\rho d \boldsymbol{\Sigma})
$$

or in the coordinates,

$$
D\left(\rho d \Sigma_{i}\right)=-\left(\rho d \Sigma_{k}\right) \partial_{i} v_{k}
$$

Now it is easy to prove relation (89) without any restrictions for the type of flow. Namely,

$$
\begin{array}{r}
D(\mathbf{M} \cdot d \boldsymbol{\Sigma})=D(\mathbf{m} \cdot \rho d \boldsymbol{\Sigma})=D \mathbf{m} \cdot \rho d \boldsymbol{\Sigma}+m_{i} D\left(\rho d \Sigma_{i}\right)= \\
\quad=\rho d \boldsymbol{\Sigma} \cdot(\mathbf{m} \cdot \nabla) \mathbf{v}+\mathbf{j} \cdot d \boldsymbol{\Sigma}-m_{i} \rho d \Sigma_{k} \partial_{i} v_{k}=\mathbf{j} \cdot d \boldsymbol{\Sigma} .
\end{array}
$$

In order to prove the helicity conservation, Eq. (76), let us consider some scalar quantity of the form

$$
Y=\mathbf{v}_{h} \cdot \mathbf{J}
$$

where $\mathbf{J}$ is some frozen-in field. Then, taking into account that Eq. 69) for the barotropic flows can be rewritten as

$$
D \mathbf{v}_{h}=-\nabla\left(\chi-v^{2} / 2\right)-v_{h m} \cdot \nabla v_{m}, \quad \chi \equiv \int d p / \rho,
$$

we obtain

$$
D Y=D \mathbf{v}_{h} \cdot \mathbf{J}+\mathbf{v}_{h} \cdot D \mathbf{J}=-\nabla\left(\chi-v^{2} / 2\right) \cdot \mathbf{J} .
$$

For $\mathbf{J}=\boldsymbol{\omega}_{h} / \rho$ we proceed

$$
D\left(\mathbf{v}_{h} \cdot \boldsymbol{\omega}_{h} / \rho\right)=-\rho^{-1}\left(\nabla\left(\chi-v^{2} / 2\right) \cdot \boldsymbol{\omega}_{h}\right)=-\rho^{-1} \operatorname{div}\left(\left(\chi-v^{2} / 2\right) \boldsymbol{\omega}_{h}\right) .
$$

Then

$$
D\left(\mathbf{v}_{h} \cdot \boldsymbol{\omega}_{h}\right)=\rho D\left(\mathbf{v}_{h} \cdot \boldsymbol{\omega}_{h} / \rho\right)+\left(\mathbf{v}_{h} \cdot \boldsymbol{\omega}_{h} / \rho\right) D \rho=-\operatorname{div}\left(\left(\chi-v^{2} / 2\right) \boldsymbol{\omega}_{h}\right)-\left(\mathbf{v}_{h} \cdot \boldsymbol{\omega}_{h}\right) \operatorname{div} \mathbf{v}
$$

or

$$
\partial_{t}\left(\mathbf{v}_{h} \cdot \boldsymbol{\omega}_{h}\right)=-\operatorname{div} \mathbf{q}_{h}, \quad \mathbf{q}_{h}=\left(\chi-v^{2} / 2\right) \boldsymbol{\omega}_{h}+\mathbf{v}\left(\mathbf{v}_{h} \cdot \boldsymbol{\omega}_{h}\right)
$$

that evidently coincides with Eq. (76).

It is noteworthy that the proof is valid for arbitrary $\mathbf{J}$ - type invariant if the field $\rho \mathbf{J}$ is divergence-free and the flow is barotropic:

$$
\partial_{t}\left(\rho \mathbf{J} \cdot \mathbf{v}_{h}\right)=-\operatorname{divq}, \quad \mathbf{q}=\left(\chi-v^{2} / 2\right) \rho \mathbf{J}+\mathbf{v}\left(\rho \mathbf{J} \cdot \mathbf{v}_{h}\right) \quad \text { for } \quad \operatorname{div}(\rho \mathbf{J})=0 .
$$

For instance, choosing $\mathbf{J}=\mathbf{h}$ immediately leads to the cross-helicity invariant if one takes into account that $\mathbf{H} \cdot \mathbf{v}_{h}=$ $\mathbf{H} \cdot \mathbf{v}$. 


\section{Acknowledgment}

This work was supported by the INTAS (Grant No. 00-00292).

[1] V. E. Zakharov, E. A. Kuznetsov, Hamiltonian formalism for nonlinear waves, Uspechi Fizicheskich Nauk (Physics Uspechi) 40, 1087-1116 (1997).

[2] V. P. Goncharov, V. I. Pavlov, The problems of hydrodynamics in Hamiltonian description, MGU Publishers, Moskow (1993). (in Russian)

[3] V. E. Zakharov, V. S. L'vov, G. Falkovich, Kolmogorov Spectra of Turbulence. Wave Turbulence, Springer-Verlag, N.Y. (1992).

[4] E. A. Kuznetsov, Weak magnetohydrodynamic turbulence of magnetized plasma, Zh. Eksp. Teor. Fiz. (Sov. Phys. JETP) 120, No 11, 1213 (2001). (in Russian)

[5] V. I. Arnold, Variational principle for a three-dimensional stationary flows of ideal fluid, Prikl. Mech. i Mathem. 29, No 5, 846-851 (1965) (in Russian); Mathematical Methods of Classical Mechanics, Springer, N.-Y. (1978).

[6] H. D. I. Abarbanel, R. Brown, Y. M. Yang, Hamiltonian formulation of inviscid flows with free boundaries, Phys. Fluids 31, 2802-2809 (1988).

[7] D. Lewis, J. Marsden, R. Montgomery, The Hamiltonian structure for hydrodynamic free boundary problems, Physica 18D, 391-404 (1986).

[8] V.A. Vladimirov, H.K. Moffatt, On General Transformations and variational Principles in Magnetohydrodynamics. Part I. Fundamental Principles., J. Fl. Mech., 283, 125-139 (1995).

[9] V. A. Vladimirov, H. K. Moffatt, K. I. Ilin, On General Transformations and variational Principles in Magnetohydrodynamics. Part II. Stability Criteria for two-dimensional Flows., J. Fl. Mech., 329, 187-205 (1996); Part III. Stability Criteria for Axisymmetric Flows., J. Plasma Phys., 57, part 1, 89-120 (1997); Part 4. Generalized Isovorticity Principle for three-dimensional Flows., J. Fl. Mech., 390 127-150 (1999).

[10] S. S. Moiseev, R. Z. Sagdeev, A. V. Tur, V. V. Yanovsky, Frozen-in integrals of motion and Lagrange invariants in the hydrodynamic models, Zh. Eksp. Teor. Fiz. (Sov. Phys. JETP) 83, No 1, 215-226 (1982) (in Russian); Problems of the theory of strong turbulence and topological solitons. In the book: "Nonlinear Phenomena in Plasma and Hydrodynamics", M., Mir publishers, 137-182 (1986).

[11] R. Z. Sagdeev, A. V. Tur, V. V. Yanovsky, Construction of Frozen-in Integrals, Lagrangian and Topological Invariants in Hydrodynamical Models, In Topological Fluid Mechanics, Cambridge Univ. Press, 421 (1990).

[12] A. V. Tur, V. V. Yanovsky, Invariants in Dissipationless Hydrodynamics media, J. Fluid. Mech. 248, No 1, $67-106$ (1993).

[13] D. V. Volkov, A. V. Tur, V. V. Yanovsky, Hidden supersymmetry of classical systems (hydrodynamics and conservation laws), Phys. Lett. A, 203, 357-361 (1995).

[14] A. V. Kats, Variational principles and canonical variables for MHD flows with breaks I, Radiofizika\&Radiostronomia, 7, No 3, 232-245 (2002). (in Russian)

[15] L. Woltjer, A theorem on force-free magnetic fields, Proc. Nat. Acad. Sci., 44, 489-491 (1958).

[16] M. Steenbeck, F. Krause, The generation of stellar and planetary magnetic fields by tubulent dynamo action, Z. Naturforsch., 21 A, 1285-1296 (1966).

[17] H. K. Moffatt, The degree of knottedness of tangled vortex' lines, J. Fluid. Mech. 35, 117-129 (1969).

[18] E. Hameiri, Phys. Plasmas 5, 3270 (1998).

[19] J. A. Almaguer, E. Hameiri, J. Herrera, and D. D. Holm, Phys. Fluids 31, 1930 (1988).

[20] V. I. Ilgisonis and V. P. Pastukhov, Physika Plasmy 22, 228 (1996). (in Russian)

[21] A. V. Kats, Variational principle in canonical variables, Weber transformation and complete set of the local integrals of motion for dissipation-free magnetohydrodynamics, JETP Lett., 77, No 12, 657-661 (2003); Canonical description of magnetohydrodynamic flows and integrals of motion, arXiv: physics/0212023 (2002)

[22] V. E. Zakharov, E. A. Kuznetsov, Variational principle and canonical variables in magnetohydrodynamics, DAN SSSR, 194, 1288 (1970). (in Russian)

[23] H. Weber. Ueber eine Transformation der hydrodynamischen Gleichungen, J. Reine Angew. Math. 68, $286-292$ (1868).

[24] H. Lamb, Hydrodynamics, Cambridge Univ. Press (1932).

[25] A. V. Kats, V. M. Kontorovich, Hamiltonian description of the motion of discontinuity surfaces, Low Temp. Phys., 23, No 1, 89-95 (1997).

[26] A. V. Kats, Variational principle and canonical variables in hydrodynamics with discontinuities, Physica D, 152-153, 459-474 (2001).

[27] P. A. M. Dirac, Lectures on quantum mechanics, Yeshiva Univ., N.Y. (1964).

[28] D. M. Gitman, I. V. Tyutin, Canonical quantization of the fields with constraints, M., Nauka, (1986). (in Russian)

[29] J. Serrin. Mathematical principles of classical fluid mechanics. Handbuch der Physik. Stromungsmechanik I, $125-262$. Springer (1959).

[30] R. Salmon. Hamiltonian principle and Ertel's theorem. In Conf. Proc. Am. Inst. Phys. 88, 127-135 (1982).

[31] V. A. Gordin, V. I. Petviashwili, Fiz. Plasmi 13, 509 (1987). (in Russian)

[32] This form of the action slightly differs from that proposed in Ref. [14]. The main difference consists in introducing the 
vector potential for the magnetic field. Therefore, here the canonical pair is $\mathbf{A},-\mathbf{M}$ instead of $\mathbf{H}, \mathbf{S}$, where $\mathbf{S}=$ curlM. We do not consider the discontinuous flows and thus we omit the surface term in the action. But adding corresponding surface term we can easily take the breaks into account.

[33] Note that substituting $\mathbf{M}=$ curlS into Eq. (11) one can integrate it and arrive at the dynamic equation for $\mathbf{S}$, $\partial_{t} \mathbf{S}=$ $(4 \pi)^{-1} \mathbf{H}+\mathbf{v} \times$ curl $\mathbf{S}+\nabla \Psi$, where $\Psi$ represents a scalar field respectful for the $\mathbf{S}$ gauge. This relation only by the $\mathbf{S}$ sign differs from equation (10.9) of the reference [1] (or Eq. (7) in the original paper 22]).

[34] We do not include $\Lambda$ into the set of canonical variables dealing with the extended Hamiltonian description, cf. Refs. 27, 28]. Otherwise, we can include $\Lambda$ into the set of generalized coordinates. Denoting corresponding conjugate momentum $\pi_{\Lambda}$ and adding to the Hamiltonian density, Eq. (18), the term $\pi_{\Lambda} \nu$ results in

$$
\partial_{t} \pi_{\Lambda}=-\delta \mathcal{H} / \delta \Lambda=-\operatorname{div} \mathbf{M}, \quad \partial_{t} \Lambda=\delta \mathcal{H} / \delta \pi_{\Lambda}=\nu .
$$

Variation of the action with respect to the additional variable $\nu$ results now in the restriction $\pi_{\Lambda}=0$. This restriction is consistent with the set of canonical equations. Namely, Eq. (11) (or, equivalently, the canonical equation $\partial_{t} \mathbf{M}=\delta H / \delta \mathbf{A}$ ) leads to $\partial_{t} \operatorname{div} \mathbf{M}=0$. The momentum $\pi_{\Lambda}$ is the linear function of $t, \pi_{\Lambda}=\pi_{\Lambda}\left(t_{0}\right)-\left(t-t_{0}\right)$ divM. The condition $\pi_{\Lambda}=0$ follows for the specific choice $\pi_{\Lambda}\left(t_{0}\right)=0$ and $\operatorname{div} \mathbf{M}\left(t_{0}\right)=0$. Moreover, as it becomes clear below, the initial condition $\mathbf{M}\left(t_{0}\right)=0$ (resulting in $\operatorname{div} \mathbf{M}\left(t_{0}\right)=\operatorname{div} \mathbf{M}(t)=0$ ) leads to essential simplifications. On the other hand, conditions $\pi_{\Lambda}=0$, $\mathbf{M}\left(t_{0}\right)=0$ do not lead to any restrictions on the physical variables and the MHD flow as well. The subsidiary functions $\Lambda$ and $\nu$ can be expressed in terms of other variables as $\Lambda=\Delta^{-1}(\operatorname{div}[\mathbf{v} \times \mathbf{H}]-\operatorname{div} \dot{\mathbf{A}})+\Lambda^{\prime}, \nu=\partial_{t} \Lambda$, where $\Delta$ denotes the Laplace operator and $\Lambda^{\prime}$ is arbitrary solution of the Laplace equation.

[35] Note that the considerations based upon the Pfaff's theorem also result in the reduced velocity representation. But this theorem in our case claims only the local equivalence between the three-dimensional vector field and the standard form $\phi+\lambda \nabla \mu$ with the appropriate scalars $\phi, \lambda, \mu$. This point is often ignored.

[36] In terms of the differential forms $\alpha$ and $\mathbf{I}$ are scalar and vector 0 -forms; $\mathbf{L}, \mathbf{J}$ and $\rho$ are $1-, 2$ - and 3-forms, respectively. 